\title{
Placement Variations and their Diagnosis
}

\author{
Waltenegus Dargie \\ Technical University of Dresden \\ Chair of Computer Networks \\ Faculty of Computer Science \\ 01062 Dresden \\ waltenegus.dargie@tu-dresden.de
}

\author{
Alexander Schill \\ Technical University of Dresden \\ Chair of Computer Networks \\ Faculty of Computer Science \\ 01062 Dresden \\ alexander.schill@tu-dresden.de
}

\begin{abstract}
This paper investigates the impact of placement and orientation variations on the quality of sensed data. Different types of human movements are considered, namely, balancing, skipping, leaping; climbing up and down a staircase, and running. For data collection, tri-axis accelerometer sensors are used. As target placements, arms, thighs, knees, ankle, and waist are considered. Likewise, four different orientation angles were considered during deployment, namely, 0 , 30,45 , and 85 degrees. The features employed to investigate placement and orientation variations were zero $\backslash$ mean-value crossing rate, correlation coefficients, cross-correlation, and auto-correlation. A particular focus was given to steady slow movements (climbing up and down a staircase) and steady fast movements (running). Remarkably, the fast movements are less affected by placement variations in comparison to the slow movements. Moreover, it will be shown that the effect of orientation variations for all types of movements are insignificant when absolute acceleration instead of the accelerations of individual axes are independently considered.
\end{abstract}

\section{ACM Classification Keywords}

C.2.4 Computer-Communication Networks: Distributed Systems_-distributed applications; H.4 Information Systems Applications: Miscellaneous

\section{General Terms}

Algorithm, measurement, analysis, experiment

\section{Author Keywords}

Context-awareness, signal processing, feature extraction, movement analysis, activity monitoring, placement, orientation

\section{INTRODUCTION}

More and more sensors are becoming integral parts of mobile devices and physical environments, enabling users to have enhanced awareness of and control over their environments. For example, sensor-based applications have been

Permission to make digital or hard copies of all or part of this work for personal or classroom use is granted without fee provided that copies are not made or distributed for profit or commercial advantage and that copies bear this notice and the full citation on the first page. To copy otherwise, or republish, to post on servers or to redistribute to lists, requires prior specific permission and/or a fee.

CASEMANS '10, Sep 26, 2010, Copenhagen, Denmark.

Copyright 2010 ACM 978-1-4503-0213-5...\$10.00. useful for industrial process monitoring and control [9], home and office automation [4], healthcare [6], object tracking and logistic as well as transportation management [15].

For some of these applications, the sensors are attached to mobile entities (humans and objects) whose activities should be monitored. For example, Marin-Perianu et al. [15] attach accelerometer sensors to containers in supply-chain management to ensure that certain items are travelling together. Similarly, Malinowski et al. [14] install accelerometer sensors on crates to monitor the safety of fragile items during transportation. Sensors have also been used in the medical profession to monitor the activities of nurses $[12,5]$ and patients [13]. In fact, there is a whole host of applications in wearable computing (for example, [1], [16], [3]) where the movements and activities of various parts of the bodies of persons are monitored.

For movement-based applications, the appropriate placement of sensors is vital to correctly capture the phenomena of interest (such as stiffness in motion or modal changes in the fundamental frequencies). This is because different parts of the body of a moving entity (a person or an object) produce or are subject to different accelerations. Displacement from the original placements or orientations of sensors can result in a significant amount of inaccuracies during signal processing. But placement and orientation variations are sometimes unavoidable on account of the movement of the objects to which the sensor nodes are attached or because of lack of knowledge on the part of the users as to the exact location of sensor placements during operation.

This paper investigates the impact of placement and orientation variations on the expressiveness of the features extracted from sensor data. Various human movements - running along a straight line, skipping, hopping, balancing, climbing up and down a staircase - were considered during the experiment. In each case, sensors were attached to the thighs, calves, ankles, wastes and arms of a person. Particular attention is given to the relatively stable movements, namely, climbing up and down a staircase (slow movements) and running (fast movement) ${ }^{1}$. Repeated experiments show that running is relatively insensitive of placement variations while the slow movements are not. Moreover, all types of movements are relatively unaffected by different orientation an-

\footnotetext{
${ }^{1}$ Sudden changes in movements can be captured and classified with comparative ease.
} 
gles when the absolue acceleration is taken into account.

The remaining part of the paper is organised as follows: in Section, the work related to sensor placement and signal processing is summarised. In Section, the methodology for investigating the impact of placement and orientation variations on the quality of sensed data is discussed in detail. In Section experiment results are analysed. Finally, in Section, concluding remarks is given.

\section{RELATED WORK}

In sensing-based applications, feature selection is a critical step, since different features can express different aspects of the measurements. However, the type of features that are selected should not be sensitive to measurement variations, among which are placement variations or nonoptimal placement of sensors.

Bao et al. [2] evaluate different machine learning algorithms to capture twenty types of movement-related human activities. The preprocessing of the raw data from accelerometer sensors is carried out at a sampling frequency of $76.25 \mathrm{~Hz}$ and a window length of 512 samples with $50 \%$ window overlap. Among the algorithms examined - decision table, C4.5 decision tree, and naive Bayes classifier -, C4.5 decision tree outperforms the rest. The study demonstrates that the recognition accuracy depends on placement of the accelerometer sensors (placement on thighs and wrists increase recognition accuracy for all movement types).

Dargie et al. [7, 8] provide quantitative and qualitative analysis of the robustness of time and frequency domain features to placement variations. The time domain features include: mean value, zero crossing rate, correlation coefficient, cross correlation and autocorrelation. The frequency domain features include: maxima, energy, linear correlation coefficient of STFT, spectral roll-off, spectral centroid and spectral flux. The measurements of human and car movements are captured by tri-axial accelerometers at $150 \mathrm{~Hz}$ synchronously. The sensor nodes are deployed randomly with different placements and orientations. In this work, zero-crossing rate is easily affected by calibration error for all types of movements while mean-crossing rate can solve this problem with low deviation. The deviation of mean value of absolute acceleration of car measurements is very small even for different orientations of sensors, but it is large for human movements. Correlation coefficients for car movements are large while they are small for human movements. Cross correlation and autocorrelation are useful when the measurements are not synchronous. Fuzzy membership functions based on empirical observations are used to qualitatively describe the uncertainties of each feature. For frequency domain features, the linear correlation coefficients of Short Time Frequency Transform outperform in roboustness all the other features.

Huynh et al. [11] analyse how recognition accuracy is affected by different features with different settings (window length) for different types of human movements. The data set used for the analysis includes six activities: walking,

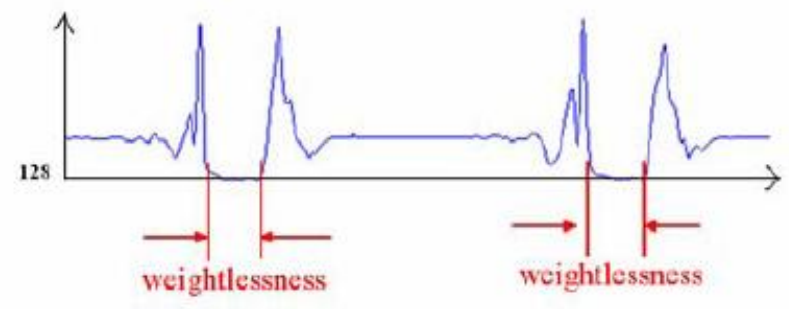

Figure 1. The weightlessness of jumping in vertical direction [10]

standing, jogging, skipping, hopping and riding bus, under unsupervised condition. The features are calculated for different window lengths, namely, 128, 256, 512, 1024 and 2048 samples with 0.25 s overlapping size. The sampling rate is $512 \mathrm{~Hz}$. Mean, variance, energy, spectral entropy and 19 additional features obtained by pair-wise addition of FFT coefficients $1+2,2+3,3+4, \ldots, 19+20$. K-means clustering is employed with five-fold cross validation for a total of 50,000 samples. The study shows that (1) there is a higher variation in recognition accuracy for activities of higher intensity; (2) FFT coefficients lead to a higher precision than other features, but there are some exceptions; (3) accuracy varies considerably between different FFT coefficients; and (4) no one FFT coefficient outperforms in all activities. For different window sizes, generally $1 \mathrm{~s}$ and $2 \mathrm{~s}$ length achieve higher accuracies, however, similar to FFT coefficient, no one window length outperforms in all activities.

In addition to the widely employed features, some novel features are investigated elsewhere. For example, He et al. [10] introduce the "weightlessness" feature for human activity recognition and demonstrate that the average accuracy is better than the ones widely used in other work. The raw data were collected from tri-axial accelerometers at a sampling frequency of $100 \mathrm{~Hz} .43$ subjects were involved in reasoning about four types of movements: jumping, still, running and walking. In order to evaluate robustness to placement variations, data were collected from various parts of a human body (chest pockets, waist bell, and trousers pockets). Two features are extracted. The first one is peak feature, which indicates the intensity of a signal and is computed based on net acceleration (absolute acceleration) after noise removing. The second one is weightlessness feature, which means, a short-time ground-leave-weightless state of the subject when she is running or jumping. The weightless feature is computed from vertical directional acceleration signals. A six-dimensional high-level feature from the above two features (the mean of the peak height, the mean of the weightlessness length, the mean of the peak interval, the mean of the weightlessness interval, the ratio of the peak number to the weightlessness number and the ratio of the weightlessness length to the window length) is then computed over 512 samples length frame with $50 \%$ overlapping size. This feature is used for the input of Support Vector Machine classifier. It could perform at an accuracy of up to $97.21 \%$.

The approaches dicussed above deal with placement varia- 
Table 1. Description of movements in experiments: the content of left column is the name of movements with abbreviation in brackets and the content of right column is the description of movements.

\begin{tabular}{|l|l|}
\hline Movement & Description \\
\hline Balancing Test (B) & $\begin{array}{l}\text { The subjects stand on right leg with } \\
\text { two arms unwind for a duration of } \\
10 \text { s }\end{array}$ \\
\hline $\begin{array}{l}\text { Running along a } \\
\text { straight line(R) }\end{array}$ & $\begin{array}{l}\text { The subjects run for about 10s along } \\
\text { a straight line at a normal speed }\end{array}$ \\
\hline Hopping(H) & $\begin{array}{l}\text { The subjects hop for about 10s in the } \\
\text { forward direction }\end{array}$ \\
\hline Skipping(S) & $\begin{array}{l}\text { The subjects skip for about 10s in } \\
\text { the forward direction }\end{array}$ \\
\hline Leaping(L) & $\begin{array}{l}\text { The subjects take off from one foot } \\
\text { and land with the opposite foot for } \\
\text { about 10s in the forward direction }\end{array}$ \\
\hline $\begin{array}{l}\text { Climbing up- } \\
\text { stairs(U) }\end{array}$ & $\begin{array}{l}\text { The subjects walk upstairs (about } 8 \\
\text { steps) at a normal speed }\end{array}$ \\
\hline $\begin{array}{l}\text { Climbing down- } \\
\text { stairs(D) }\end{array}$ & $\begin{array}{l}\text { The subjects walk downstairs (about } \\
8 \text { steps) at a normal speed }\end{array}$ \\
\hline
\end{tabular}

tions only implictly. This work attempts to explicitly analyse the impact of placement on the quality of sensed data and the expressiveness of the features that should deal with imperfect sensing.

\section{METHODOLOGY}

The purpose of this paper is to investigate how placement of sensors can affect the quality of sensed data and the expressiveness of the features that are extracted from the raw data. Different types of activities (summarised in Table 1) were considered during the experiment. For each activity, measurements were taken from different parts of five healthy subjects using tri-axis accelerometers in a synchronised manner. The targeted placements were thighs (the point of reference), knees, calves, ankles, arms, and waist; at different orientations, namely, $0,30,45$, and 85 degrees. The $x-y$ plane is parallel to the surface of the body; and the y-axis is the vertical axis. Each time, measurements were taken in pair, as thigh-thigh, thigh-ankle, thigh-calf, etc. The data were sampled at a frequency of $100 \mathrm{~Hz}$ and the duration of each activity was on average $1.5 \mathrm{~s}$. Altogether, 840 pairs of data sets were gathered and analysed.

Even though a large number of time and frequency domain features were evaluated, only three of them will be discussed here - These are zero \mean-crossing rate, correlation coefficients, cross correlation, and autocorrelation.

\section{Absolute Acceleration}

The simplest way to deal with orientation variation is to compute the absolute acceleration before preprocessing instead of considering the acceleration of individual axes of a tri-axial accelerometer. The absolute acceleration is given as:

$$
a b s=\sqrt{x^{2}+y^{2}+z^{2}}
$$

\section{Zero $\backslash$ Mean-Crossing Rate}

Zero-crossing rate is commonly used for noise detection in digital signal processing, especially in audio signal processing. It expresses the frequency at which a signal crosses a zero-reference line. However, zero-crossing rate has its limitation. First, the reference line is fixed, so the value is not robust when the measurements are corrupted by uncertainties, for instance, when they are lifted up or down by calibration error. Second, the zero-crossing rate will be always zero and make no sense if the measured values are all larger than zero. Therefore, in most cases, it is usually substituted by mean-crossing rate, which is also adopted here. Similar to zero-crossing rate, mean-crossing rate can capture characteristics such as the frequency in temporal structure of a movement. The mean-value crossing rate is described as follows:

$$
\operatorname{MCR}(X)=\frac{1}{f} \sum_{i=1}^{n-1} F\left(\left(x_{i}-\bar{X}\right) \cdot\left(x_{i+1}-\bar{X}\right)\right)
$$

where $f$ is the number of frames. $F=1$ if $\left(x_{i}-\bar{X}\right) \cdot\left(x_{i+1}-\right.$ $\bar{X})<0$ and $F=0$ if $\left(x_{i}-\bar{X}\right) \cdot\left(x_{i+1}-\bar{X}\right) \geq 0$.

\section{Correlation Coefficients}

In digital signal processing, correlation coefficients are useful statistics to express the relationship between two data sets. The absolute values indicate the degree of the correlation while the sign indicates the direction of dependency. The range of values is between -1 and 1 . The closer an absolute value to 1 , the higher is the degree of relation. A negative sign indicates an inverse relationship. It is computed as:

$$
\begin{aligned}
\rho_{X, Y} & =\frac{\operatorname{Cov}(X, Y)}{\operatorname{StdX} X \operatorname{Std}_{Y}} \\
& =\frac{\sum_{i=1}^{n}\left(x_{i}-\bar{X}\right)\left(y_{i}-\bar{Y}\right)}{\sqrt{\sum_{i=1}^{n}\left(x_{i}-\bar{X}\right)^{2}} \sqrt{\sum_{i=1}^{n}\left(y_{i}-\bar{Y}\right)^{2}}}
\end{aligned}
$$

where $\operatorname{Std}(X)$ and $\operatorname{Std}(Y)$ are the standard deviation of the two data sets $X$ and $Y ; \operatorname{Cov}(X, Y)$ is the covariance between $X$ and $Y$. As shown in equation 3, the correlation coefficients are computed pair by pair, indicating the need for time synchronization during data collection. With correlation coefficients it is possible to examine a relationship without being directly dependent on local variations in amplitude.

$\rho_{X, Y}=\frac{N \sum_{i=1}^{n} x_{i} y_{i}-\sum_{i=1}^{n} x_{i} \sum_{i=1}^{n} y_{i}}{\sqrt{N \sum_{i=1}^{n} x_{i}^{2}-\left(\sum_{i=1}^{n} x_{i}\right)^{2}} \sqrt{N \sum_{i=1}^{n} y_{i}^{2}-\left(\sum_{i=1}^{n} y_{i}\right)^{2}}}(4)$

\section{Cross-Correlation}

A cross-correlation is useful to examine the existence of correlation even when the two data sets cannot be compared pair by pair. This can happen, for instance, if the data sets are 
not time-synchronised or have different sample sizes. Crosscorrelation compares the two data sets in different time-lagged instances:

$$
\gamma_{x y}(d)=\sum_{i=0}^{N-1} x_{i} \cdot y_{i-d}
$$

If the index of $y$ is less than 0 , the common approach is to assume that the value is zero for $i-d \leq 0$ or use the circular cross-correlation. This paper adopts the second approach. Hence, when $i-d \leq 0, y_{i+n-d}$ is used to replace $y_{i-d}$. Consequently, Equation 6 results in a sequence having $n$ length with a delay that ranges between $[0, \mathrm{n}-1]$. For each delay, the higher the sum of the correlation value, the higher the degree of correlation between the two data sets.

\section{ANALYSIS}

Due to space limitation, only two types of activities are considered for a detailed analysis. These are climbing up and down a staircase as steady slow movements and running as a steady fast movement. The mean value crossing rate of the slow movements are relatively agnostic of placement variations. This does not mean, however, they are expressive. In fact, it is not possible to distinguish between climbing up and climbing down by simply considering the mean value rates. On the other hand, the peak-to-peak variations of the cross correlations of slow movements are significantly larger than the corresponding values of the fast movement. Moreover, the cross correlation of the fast movement consistently exhibit a discernable periodicity, which is not the case with the slow movements.

The correlation between the left and right thighs (thigh-thigh) was not as remarkable as expected. Whereas in both types of movement there should be correlation between the left and right tigh, in almost all the cases, this was rarely outstanding. In fact, other body parts (for instance, thigh-waist as in the cross correlation of climbing upstairs or thigh-calf as in the cross correlation of running) exhibit strong correlation by comparison.

The mean-value crossing rate of the fast movement is extremely placement dependent. The variation between the different pairs of measurements is between 5 and 15/s whereas the maximum variation for the slow movements is $4 / \mathrm{s}$. Likewise, the correlation coefficients are strongly placement dependent for all types of movements. Not only were their values very small even for the correlated measurements, but also the deviation between the different pairs of measurements were high.

As expected, orientation variations do not have significant effect for all types of movements, since in the experiment the absolute acceleration instead of the individual accelerations is considered.

\section{CONCLUSION}

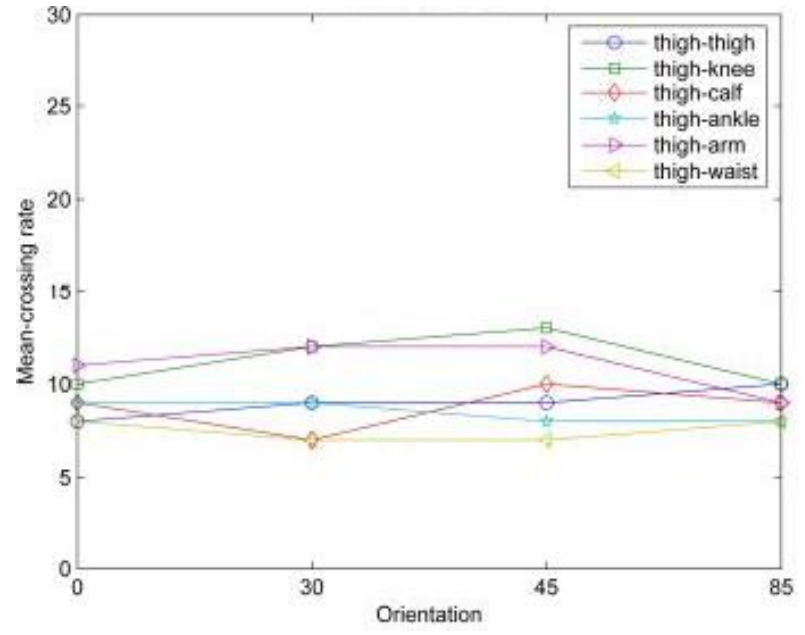

Figure 2. Mean-crossing rate of climbing upstairs with different placement and orientations

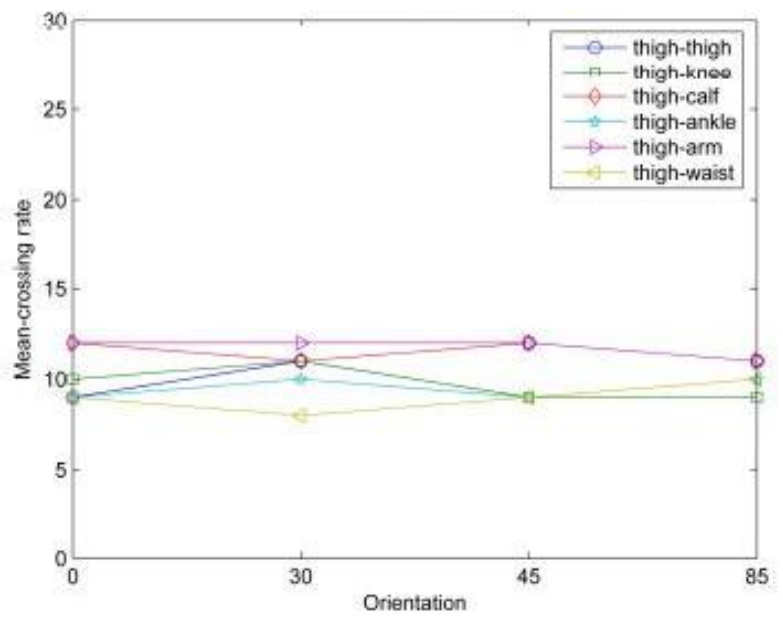

Figure 3. Mean-crossing rate of climbing downstairs with different placements and orientations

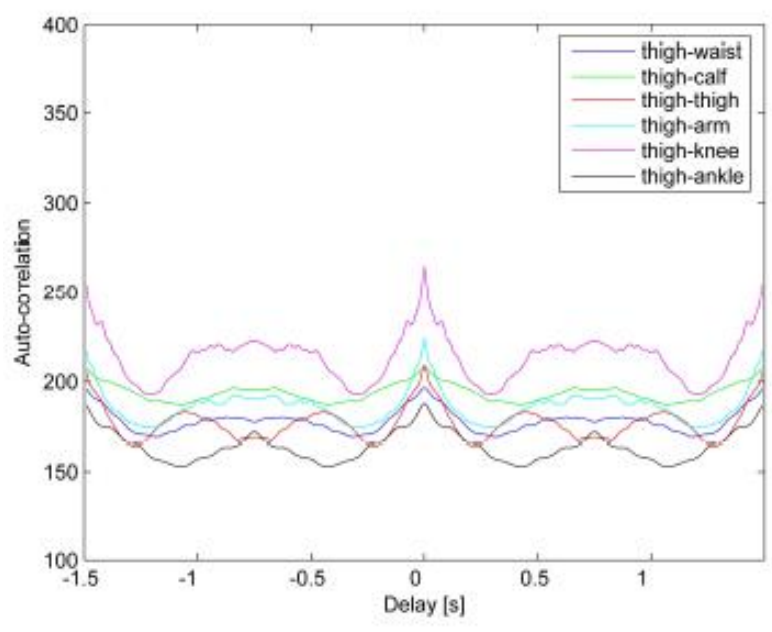

Figure 4. Cross-correlation of climbing upstairs with different placement at an orientation of $0^{\circ}$. 


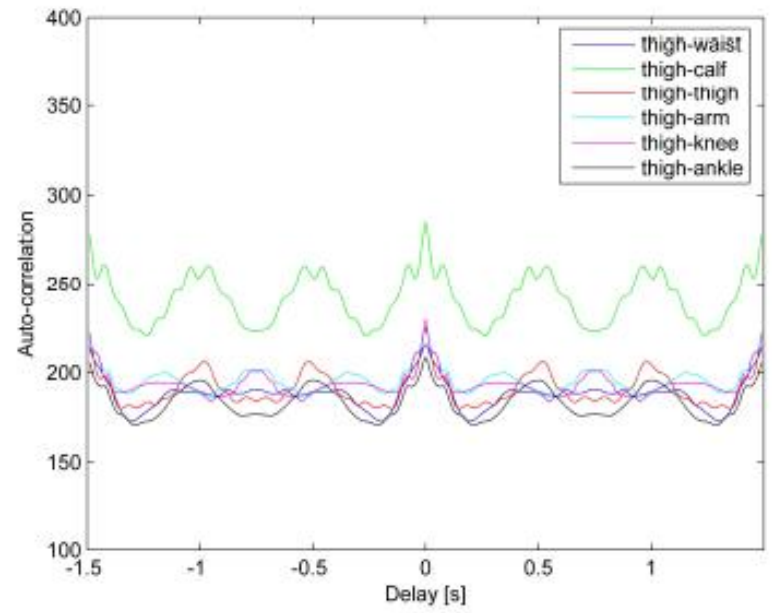

Figure 5. Cross-correlation of climbing downstairs with different placement at an orientation of $0^{\circ}$.

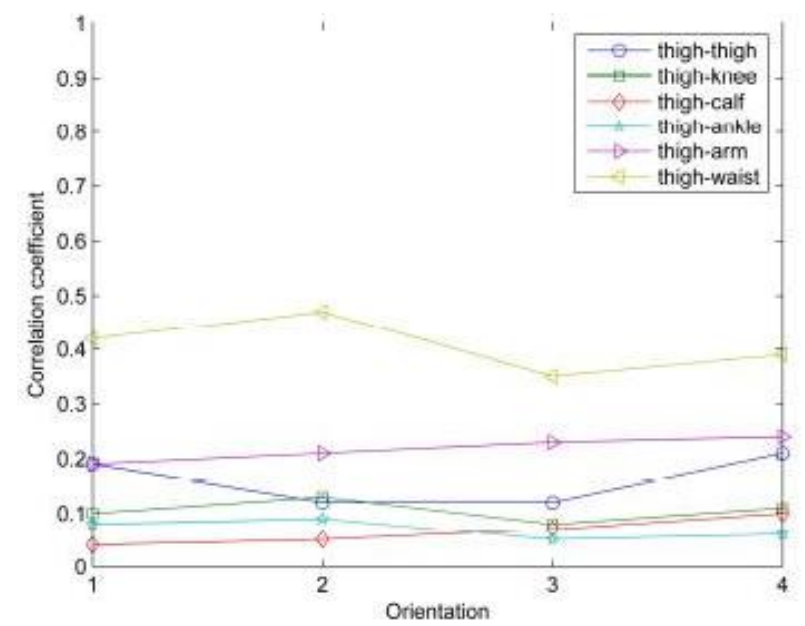

Figure 6. Correlation coefficients of climbing upstairs with different placements and orientations

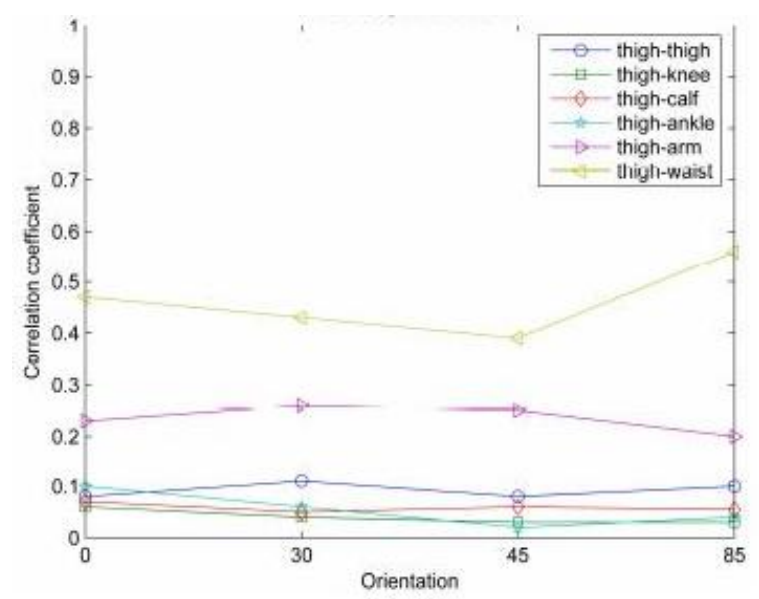

Figure 7. Correlation coefficients of climbing downstairs with different placements and orientations

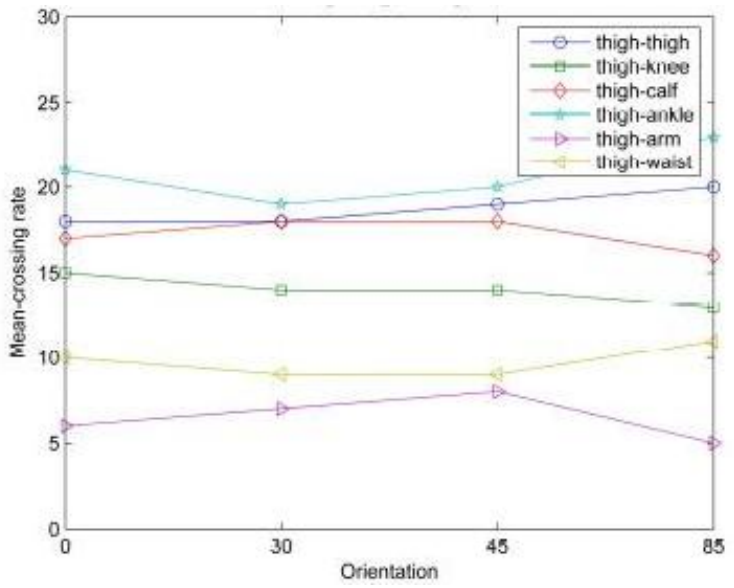

Figure 8. Mean-crossing rate of running with different placements and orientations

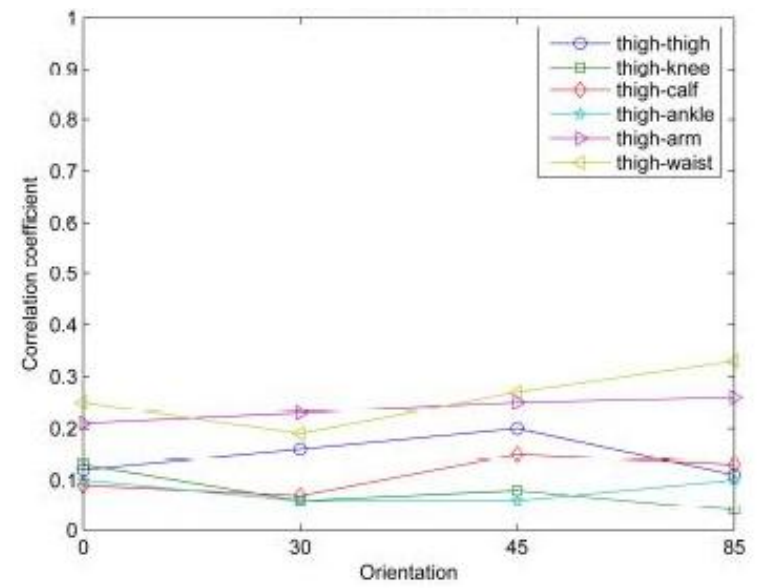

Figure 9. Correlation coefficients of running with different placements and orientations

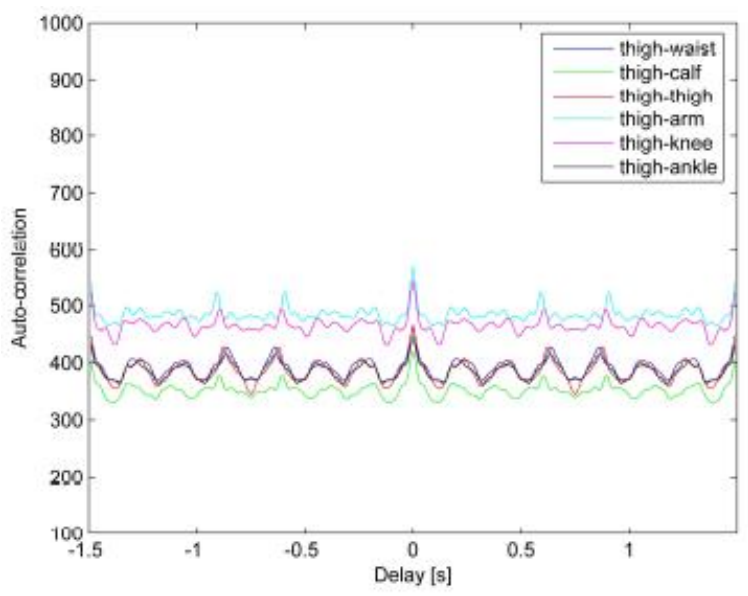

Figure 10. Cross-correlation of running with different placements at an orientation of $0^{\circ}$. 
Various types of placements and orientations were considered to investigate their impact on the quality sensed data and the expressiveness of the features that are extracted from the data. In this paper, the characteristics of two types of movements were considered with particular detail. Whereas the mean value crossing rate of the slow movements are relatively agnostic to placement variations, the feature itself is inexpressive. It has been found out that correlation coefficients the most significantly afftected by placement errors. This comes as a surprise, since these features should have not been affected by variations in the direction of movements. In all the movement types we concidered, comparison of the features taken from similar placements (thighthigh, for example) did not result in high correlation coefficients.

On the other hand, cross correlation and autocorrelation are both expressive and less sensitive to placement variations in fast movements.

\section{Acknowledgement}

The authors would like to thank Ms. Xianwen Zhang for carrying out a part of the experiment presented in this paper.

\section{REFERENCES}

1. C. Baber, D. J. Haniff, and S. I. Woolley. Contrasting paradigms for the development of wearable computers. IBM Syst. J., 38(4):551-565, 1999.

2. L. Bao and S. S. Intille. Activity recognition from user-annotated acceleration data. pages 1-17. Springer, 2004.

3. A. Bulling, D. Roggen, and G. Tröster. Wearable eog goggles: Seamless sensing and context-awareness in everyday environments. J. Ambient Intell. Smart Environ., 1(2):157-171, 2009.

4. M. Chan, D. Estève, C. Escriba, and E. Campo. A review of smart homes-present state and future challenges. Comput. Methods Prog. Biomed., 91(1):55-81, 2008.

5. M. Cheng, M. Kanai-Pak, N. Kuwahara, H. I. Ozaku, K. Kogure, and J. Ota. Dynamic scheduling based inpatient nursing support: applicability evaluation by laboratory experiments. In Casemans '09: Proceedings of the 3rd ACM International Workshop on Context-Awareness for Self-Managing Systems, pages 48-54, New York, NY, USA, 2009. ACM.

6. S. Dagtas, Y. Natchetoi, and H. Wu. An integrated wireless sensing and mobile processing architecture for assisted living and healthcare applications. In HealthNet '07: Proceedings of the 1st ACM SIGMOBILE international workshop on Systems and networking support for healthcare and assisted living environments, pages 70-72, New York, NY, USA, 2007. ACM.

7. W. Dargie. Analysis of time and frequency domain features of accelerometer measurements. In ICCCN
'09: Proceedings of the 2009 Proceedings of 18th International Conference on Computer

Communications and Networks, pages 1-6,

Washington, DC, USA, 2009. IEEE Computer Society.

8. W. Dargie and M. K. Denko. Analysis of error-agnostic time- and frequency-domain features extracted from measurements of 3-d accelerometer sensors. IEEE Systems Journal, 4:26-33, Mar. 2010.

9. L. Fortuna, S. Graziani, and A. Rizzo. Soft Sensors for Monitoring and Control of Industrial Processes (Advances in Industrial Control). Springer-Verlag New York, Inc., Secaucus, NJ, USA, 2006.

10. Z. He, Z. Liu, L. Jin, L.-X. Zhen, and J.-C. Huang. Weightlessness feature - a novel feature for single tri-axial accelerometer based activity recognition. In ICPR, pages 1-4, 2008.

11. T. Huynh and B. Schiele. Analyzing features for activity recognition. In sOc-EUSAI '05: Proceedings of the 2005 joint conference on Smart objects and ambient intelligence, pages 159-163, New York, NY, USA, 2005. ACM.

12. T. Inomata, F. Naya, N. Kuwahara, F. Hattori, and K. Kogure. Activity recognition from interactions with objects using dynamic bayesian network. In Casemans '09: Proceedings of the 3rd ACM International Workshop on Context-Awareness for Self-Managing Systems, pages 39-42, New York, NY, USA, 2009. ACM.

13. K. Lorincz, B.-r. Chen, G. W. Challen, A. R. Chowdhury, S. Patel, P. Bonato, and M. Welsh. Mercury: a wearable sensor network platform for high-fidelity motion analysis. In SenSys '09: Proceedings of the 7th ACM Conference on Embedded Networked Sensor Systems, pages 183-196, New York, NY, USA, 2009. ACM.

14. M. Malinowski, M. Moskwa, M. Feldmeier, M. Laibowitz, and J. A. Paradiso. Cargonet: a low-cost micropower sensor node exploiting quasi-passive wakeup for adaptive asychronous monitoring of exceptional events. In SenSys '07: Proceedings of the 5th international conference on Embedded networked sensor systems, pages 145-159, New York, NY, USA, 2007. ACM.

15. R. Marin-Perianu, M. Marin-Perianu, P. J. M. Havinga, and H. Scholten. Movement-based group awareness with wireless sensor networks. In Pervasive, pages 298-315, 2007.

16. W. W. Mayol-Cuevas, B. J. Tordoff, and D. W. Murray. On the choice and placement of wearable vision sensors. Trans. Sys. Man Cyber. Part A, 39(2):414-425, 2009. 